\title{
Palm Forest Landscape in Castillos (Rocha, Uruguay): Contributions to the Design of a Conservation Area
}

\section{Mercedes Rivas $^{1 *}$, José María Filippini², Henrique Cunha², Juan Hernández ${ }^{3}$, Yuri Resnichenko³, Rosa Lia Barbieri²}

${ }^{1}$ Faculty of Agronomy and Regional Eastern University Center, University of the Republic, Rocha, Uruguay

${ }^{2}$ Embrapa Temperate Climate, Pelotas, Brazil

${ }^{3}$ Faculty of Science, University of the Republic, Montevideo, Uruguay

Email: *mrivas@cure.edu.uy

How to cite this paper: Rivas, M., Filippini, J. M., Cunha, H., Hernández, J., Resnichenko, Y., \& Barbieri, R. L. (2017). Palm Forest Landscape in Castillos (Rocha, Uruguay): Contributions to the Design of a Conservation Area. Open Journal of Forestry, 7, 97-120.

https://doi.org/10.4236/ojf.2017.72007

Received: December 15, 2016

Accepted: March 17, 2017

Published: March 20, 2017

Copyright $\odot 2017$ by authors and Scientific Research Publishing Inc. This work is licensed under the Creative Commons Attribution International License (CC BY 4.0).

http://creativecommons.org/licenses/by/4.0/

(c) $\underset{\mathrm{EY}}{(7)}$ Open Access

\begin{abstract}
Butia palm forests are considered unique due to their aesthetic value, high biodiversity level and historical, archaeological and cultural value. The lack of regeneration of butia palms caused by cattle overgrazing and natural grasslands replacement by agriculture endangers these palm forests. The aim of this work is to provide information for the proposal of a conservation area in this rural landscape within the framework of sustainable development. This work was developed within the context of a Geographic Information System with thematic information on palm forest density levels, soils, land use aptitude and rural registers. Field surveys were conducted to record the presence of plant species and genetic resources in different vegetation units. The main category of soil cover was natural grasslands, followed by native forests, wetlands and palm forests. Palm forests grow mainly on soil units with poor to rather poor drainage and on arable or non-arable lands under special conditions. We identified 212 farms where the palm forest is found, a high diversity of vegetation units, the presence of 302 native taxa of plants and a significant number of plant genetic resources for various uses. For the designation of a conservation area, we propose 20 priority farms that will ensure that palm forests are well represented regarding ecosystem diversity. We consider the creation of a Protected Landscape, a State Park or a GIAHS to be the best alternatives so as to protect this unique multifunctional landscape.
\end{abstract}

\section{Keywords}

Butia odorata, In Situ Conservation, Geographic Information Systems, Plant Genetic Resources 


\section{Introduction}

Butia palms (Butia odorata (Barb. Rodr.) Noblick) are without a doubt a plant genetic resource with multiple uses that have shaped a biocultural and multifunctional landscape since pre-historical times (López Mazz \& Pintos, 2001; Dabezies, 2011; Rivas \& Barbieri, 2015), in the sense proposed by Agnoletti \& Rotherham (2015). Butia odorata (butia) palm forests form a landscape characterized by the presence of butia palms with density levels of between 50 and 600 palms per hectare over natural herbaceous grasslands. These forests are found exclusively in the Pampa biome, in the south of Brazil and Uruguay. In the Department of Rocha (Uruguay is subdivided in 19 administrative regions known as departments), in the southeast of Uruguay, two large areas of palm forests are found: the palm forests of Castillos and San Luis, which cover an area of almost 70,000 hectares (Rivas, 2005). These palm forests are considered to be unique because of their landscape and biodiversity value, and also due to the associated historical, archaeological and cultural values (PROBIDES, 1995; López Mazz \& Pintos, 2001; Geymonat \& Rocha, 2009; Rivas \& Barbieri, 2015). The Castillos palm forest is located in the middle plains of the Laguna Negra (Black Lagoon) basin, an area characterized by high environmental heterogeneity as a result of a topographic gradient from the hills to the lowlands (PROBIDES, 1999). This territory is part of the Biosphere Reserve of Bañados del Este (Eastern Wetlands) (MAB-UNESCO), with the presence of forests and bushes, natural fields or grasslands with varied physiognomies and a rich botanical composition as well as wetlands and coastal vegetation (PROBIDES, 1999; Pezzani, 2007).

While in Uruguay, the destruction of natural palm forests is forbidden (Law No. 9872 of 1939, as amended by Law No. 15,939 of 1987), this does not ensure their conservation. Most palms of the forest are more than two or three hundred years old, and there are almost no young plants to ensure the forest's survival. The lack of regeneration poses a serious threat to their preservation. These processes take place in lands which are owned exclusively by private owners for agricultural use. In the Castillos palm forest, the main activity is cattle farming on natural grasslands and, more recently, extensive agriculture. Overgrazing is a common practice in which cattle often eats and crushes young butia seedlings, causing their death. On the other hand, agriculture prevents seeds from germinating and seedlings from growing (Rivas, 2005; Rivas, 2013).

The Guidelines for Land Use Planning and Sustainable Development of the Government of Rocha state that "the aim of the development policy is to achieve a virtuous link between conservation and sustainable use of environmental values". In particular, "the conservation of palm forests in a production environment that makes its reproduction viable" is quoted as an expected result, while "promoting conservation actions for palm forests within productive land uses" appears as one of the plan's main actions.

In this sense, the concept of in situ conservation (United Nations, 1992, 2002; FAO, 1996, 2012) applies to the butia palm forest landscape. The framework for the establishment of a conservation area and the design of a management plan is 
that of sustainable use of biodiversity and their associated traditional knowledge (Hawkes et al., 1997; Maxted et al., 1997; Perrino et al., 2006; Iriondo et al., 2008; Rivas et al., 2010; Rajpurohit \& Jhang 2015; Rivas \& Condón, 2016). On the other hand, the multifunctional approach on landscapes (Cullotta \& Maetzke, 2009; Taylor et al., 2010; Reyers et al., 2012; Dale et al., 2013) takes into consideration the advantages of landscape heterogeneity and allows us to meet specific ecological, productive and cultural objectives in order to improve the condition of the land and the quality of life of its inhabitants (Taylor et al., 2010, Brussaard et al., 2010).

The overall objective of this study is to contribute with recommendations for the establishment of a conservation area and the formulation of a management plan that helps to preserve butia forests within the context of a multifunctional rural landscape. The specific objectives are to identify and quantify the various categories of land cover, to survey plant communities, species and plant genetic resources, the characterization of the environmental conditions in which palm forests are found and their distribution within commercial farms. GIS (Geographic Information Systems) and ecogeographic surveys (Guarino, 1995; Magos Brehm et al., 2008; Parra-Quijano et al., 2012; Berlingeri \& Crespo, 2012) are seen as appropriate tools so as to develop these objectives.

\section{Materials and Methods}

\subsection{Area under Study}

The studied territory was defined based on the distribution of the Castillos palm forest (Rocha, Uruguay) and its area of influence (Figure 1). The coordinates of the area are: $33^{\circ} 53^{\prime} 14.29^{\prime \prime} \mathrm{S}, 53^{\circ} 59^{\prime} 14.69^{\prime \prime} \mathrm{W}$-northwest; $33^{\circ} 52^{\prime} 38.67^{\prime \prime S}$, $53^{\circ} 36^{\prime} 25.65^{\prime \prime} \mathrm{W}$-northeast; $34^{\circ} 23^{\prime} 28.65^{\prime \prime} \mathrm{S}, 53^{\circ} 58^{\prime} 10.05^{\prime \prime} \mathrm{W}$-southwest and $34^{\circ} 22^{\prime} 52.36^{\prime \prime} \mathrm{S}, 53^{\circ} 35^{\prime} 12.89^{\prime \prime} \mathrm{W}$-southeast. It is bounded by the Atlantic Ocean on the south, by the Laguna Negra (Black Lagoon) on the east, by the Laguna de Castillos (Castillos Lagoon) on the west and by Sierra de La Blanqueada on the north.

\subsection{Methodology for the Analysis of Thematic Information}

A map produced by Zaffaroni et al. (2005) which states the palm density levels of the Castillos palm forest was used as base material. Five different density categories of the palm forest were determined by aerial photographic interpretation and field surveys (Figure 2). By using this methodology, an area of 11,611 hectares of palm forest was calculated and distributed according to the different density categories (Table 1).

The layers of digital information related to soil units and types, drainage, land aptitude and land cover were obtained from the database of the Department of Natural Resources of the Ministry of Farming, Agriculture and Fisheries of Uruguay (MGAP, 2013).

The classification of soil cover was carried out according to the Land Cover Classification System (LCCS FAO) using Landsat 5 TM 2007/2008 images of the whole country. It contains 45 classes including urban centers, water bodies, sand 


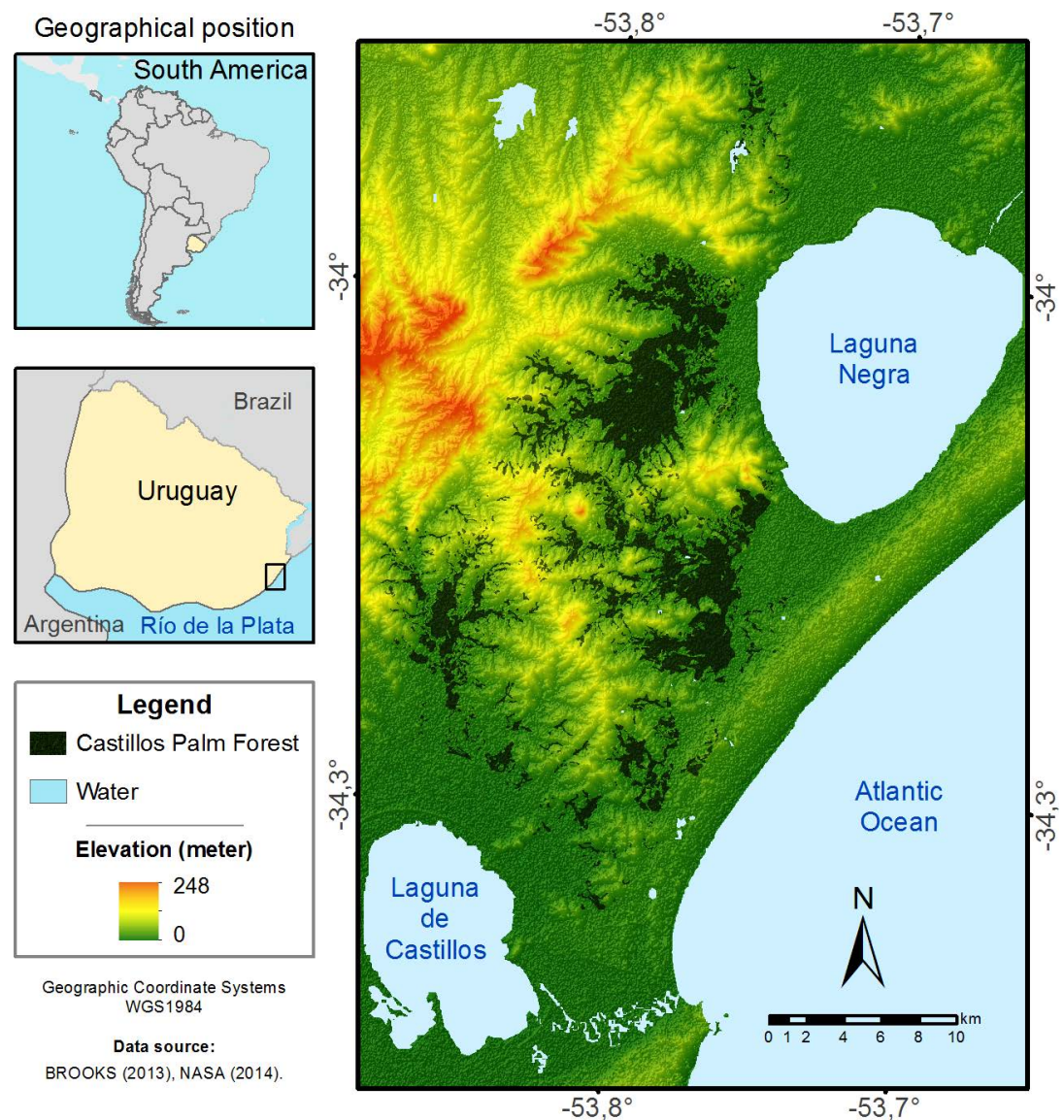

Figure 1. Map of South America, Uruguay and the Department of Rocha (left). Delimitation of the area of the Castillos palm forest (Rocha, Uruguay).
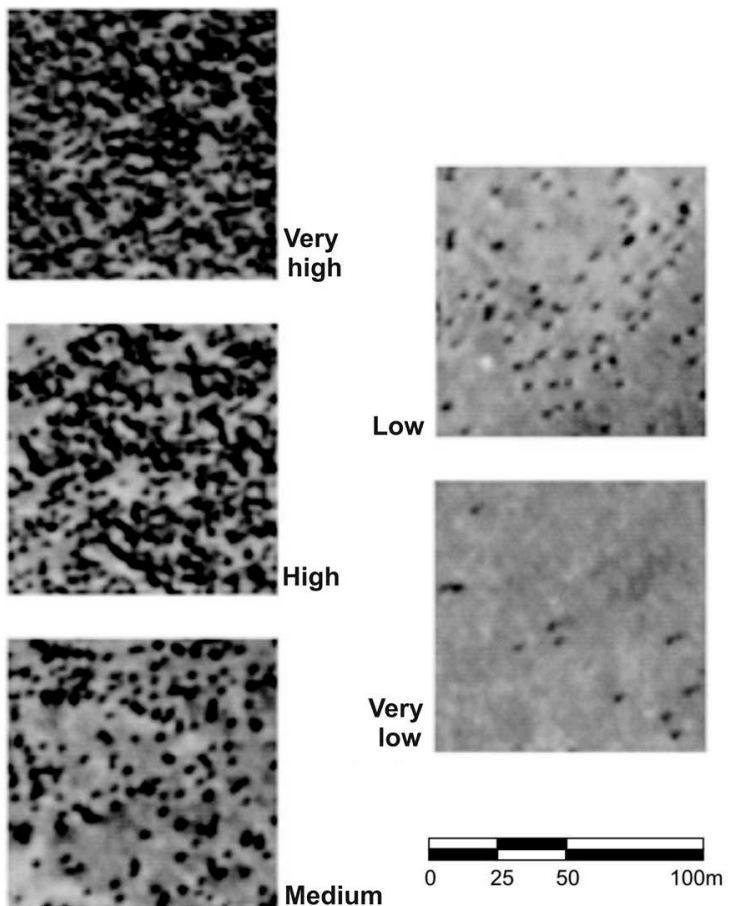

Figure 2. Density categories of the Castillos palm forest (Rocha, Uruguay) by photo-interpretation, according to Zaffaroni et al. (2005). 
Table 1. Palm forest density categories and their respective areas in Castillos (Rocha, Uruguay), according to Zaffaroni et al. (2005).

\begin{tabular}{ccc}
\hline Density category & Area (ha) & Area (\%) \\
\hline Very high (>351 palms/ha) & 223.4 & $1.9 \%$ \\
High (251 - 350 palms/ha) & 518.5 & $4.5 \%$ \\
Medium (151 - 250 palms/ha) & 1458.2 & $12.6 \%$ \\
Low (51 - 150 palms/ha) & 3890.1 & $33.5 \%$ \\
Very low (<50 palms/ha) & 5520.2 & $47.5 \%$ \\
Total & 11,611 & $100 \%$ \\
\hline
\end{tabular}

and rock formations, infrastructure and both cultivated and natural vegetation. These are standard procedures for digital processing in land management (Filippini et al., 2012). These levels of information were included in the GIS environment by cropping the portion corresponding to the area of study.

The "palm forest" class of the land cover layer was replaced by the "palm forest density classes" layer (Zaffaroni et al., 2005). Due to the different scales of the soil cover map $(1: 1,000,000)$ and the palm forest density layer $(1: 20,000)$, a surplus area was generated which was transformed into the "natural grassland" category. The resulting map included 13 soil cover classes: (1) infrastructure and urban areas, (2) water bodies, (3) wetlands, (4) natural grasslands, (5) rocky outcrops and quarries, (6) native forest, (7) cultivated forest, (8) sand formations, (9) very low density palm forest, (10) low density palm forest, (11) medium density palm forest, (12) high density palm forest; (13) very high density palm forest.

A vector layer of rural lots (basic administrative units in which the rural territory is organized) was overlapped (IDEuy, 2008). The area occupied by each class of land use in each lot was calculated using GIS.

As farms may be made up of one or more lots and this information is not officially digitalized in Uruguay, it was necessary to crosscheck the information on rural lots with registers from owners and tenants who manage cattle per lot from de Ministry of Farming, Agriculture and Fisheries. Registry units registered under the same name were grouped to form the different farms. This procedure allowed us to calculate the total area of each farm and of each land cover class in each farm.

During the springs of 2006 and 2007, seven surveys that lasted from 2 to 3 days were conducted in situ with the aim of mapping and geo-referencing vegetation units and other points of interest, using as support the 1:50,000 topographic maps and aerial photos at scale 1:20,000 obtained from the Military Geographic Service. Landscapes and plant communities were described by physiognomy. Samples of plant species of natural grasslands were obtained by using 50-meter transects every 50 centimeters. In the case of forests, wetlands and rocky outcrops, the most conspicuous species were recorded. Herbarium collections were made and photographs were taken for those cases in which it was ne- 
cessary to make laboratory observations and use keys for correct identification. The correct names of the species were verified according to the Catalog of Vascular Plants of the Southern Cone (2016). 150 sites were sampled and the average size was of about one hectare. Subsequently, using the list of plant species that had been identified, wild plant genetic resources in the landscape of the palm forest were determined by literature review.

\section{Results and Discussion}

\subsection{Soil Cover and Environmental Conditions in Which Palm Forests Are Found}

The total area studied was of 197,107.43 hectares, 66,011.64 hectares (33.49\%) of which corresponded to water bodies and 883.34 hectares $(0.45 \%)$ to urban areas and infrastructure. The distribution of the remaining land cover categories identified and their area is presented in Table 2.

The overlapping of the layer of palm forest density with those corresponding to soil units and soil types, drainage and land use aptitude, led to the conclusion that most of the palm forest area is found on the San Luis (49.03\%) and José Pedro Varela (31.18\%) soil units, as identified in the chart of soil identification of Uruguay (MGAP, 1976). These units correspond to Typic Argiaquolls (49.03\%) and Typic Argiudolls associated to Pachic Vertic Argiudolls (32.37\%), with poor to rather poor drainage. With regard to their use aptitude, they are comprised within the category of arable land under special conditions (57.76\%) or non-arable land, suitable for the production of pastures and with strong limitations for forest use (27.01\%). The distribution of the different density levels within the palm forest was not associated to soil types or use aptitude.

\subsection{Plant Community Diversity, Species and Plant Genetic Resources}

In the set of 150 surveyed sites on field trips we recorded a total of 302 plant taxa, distributed in 87 families and 224 genera (Table 3). As the identification was made only at the genus level in some cases, the number of taxa is not equiv-

Table 2. Land cover categories and their respective areas in the palm forest territory of Castillos (Rocha, Uruguay).

\begin{tabular}{ccc}
\hline Land cover category & Area (has) & Area (\%) \\
\hline Sand & 3834.03 & $2.90 \%$ \\
Wetlands & 16,431 & $12.60 \%$ \\
Natural grasslands (campos) & $66,149.74$ & $50.80 \%$ \\
Rocky outcrops and quarries & 724.34 & $0.60 \%$ \\
Native forests & $19,935.27$ & $15.30 \%$ \\
Cultivated forests & $11,523.36$ & $8.80 \%$ \\
Palm forest & $11,614.72$ & $8.90 \%$ \\
TOTAL & $130,212.46$ & $100 \%$ \\
\hline
\end{tabular}


Table 3. Plant native taxa, priority species for conservation and plant genetic resources surveyed in the palm forest territory of Castillos.

\begin{tabular}{|c|c|c|c|c|}
\hline Family & Species & Environment & $\begin{array}{l}\text { Priority for } \\
\text { conservation }\end{array}$ & $\begin{array}{c}\text { Genetic } \\
\text { Resources }\end{array}$ \\
\hline Fabaceae & Acacia bonariensis Gillies ex Hook. \& Arn. & 1 & & M \\
\hline Asteraceae & Acanthospermum australe (Loefl.) Kuntze & 4 & & OR \\
\hline Myrtaceae & Acca sellowiana (O. Berg) Burret & 1 & & F-M-OR \\
\hline Asteraceae & Achyrocline alata (Kunth) DC. & 2 & & M-OR \\
\hline Asteraceae & Achyrocline flaccida (Weinm.) DC. & 1.4 & & M \\
\hline Asteraceae & Achyrocline satureioides (Lam.) DC. & 1.4 & & M-OR \\
\hline Fabaceae & Adesmia bicolor (Poir.) DC. & 1 & & FO \\
\hline Fabaceae & Adesmia latifolia (Spreng.) Vogel & 2 & & FO \\
\hline Pteridaceae & Adiantum digitatum Hook. & 1 & & M-OR \\
\hline Orobanchaceae & Agalinis communis (Cham. \& Schltdl.) D’Arcy & 1 & & OR \\
\hline Sapindaceae & $\begin{array}{l}\text { Allophylus edulis (A. St.-Hil., A. Juss. \& Cambess.) Hieron. } \\
\text { ex Niederl. }\end{array}$ & 1 & & F-M-OR-T \\
\hline Verbenaceae & Aloysia gratissima (Gillies \& Hook.) Tronc. & 1 & & M-OR \\
\hline Amaranthaceae & Alternanthera philoxeroides (Mart.) Griseb. & 2 & & $\mathrm{M}-\mathrm{T}$ \\
\hline Asteraceae & Ambrosia tenuifolia & 3 & & M-OR \\
\hline Violaceae & Anchietea pyrifolia (Mart.) G. Don & 1 & & M \\
\hline Poaceae & Andropogon lateralis Nees & 1 & & OR \\
\hline Cyperaceae & Androtrichum trigynum (Spreng.) H. Pfeiff. & 2 & & OR \\
\hline Poaceae & Aristida sp. & 3 & & OR \\
\hline Apocynaceae & Asclepias mellodora A. St.-Hil. & 1 & & M \\
\hline Asteraceae & Aspilia montevidensis (Spreng.) Kuntze & 1.3 & & OR \\
\hline Poaceae & Axonopus fissifolius (Raddi) Kuhlm. & 3 & & $\mathrm{FO}$ \\
\hline Salicaceae & Azara uruguayensis (Speg.) Sleumer & 1 & & OR \\
\hline Salviniaceae & Azolla filiculoides Lam. & 2 & & OR \\
\hline Asteraceae & Baccharis aliena (Spreng.) Joch. Müll. & 1 & & OR \\
\hline Asteraceae & Baccharis articulata (Lam.) Pers. & 3 & & M-OR \\
\hline Asteraceae & Baccharis dracunculifolia DC. & 1 & & OR \\
\hline Asteraceae & Baccharis gnaphalioides Spreng. & 4 & & OR \\
\hline Asteraceae & Baccharis patens Baker & 1 & & $\mathrm{M}$ \\
\hline Asteraceae & Baccharis punctulata DC. & 2 & & OR \\
\hline Asteraceae & Baccharis spicata (Lam.) Baill. & 3 & & OR \\
\hline Asteraceae & Baccharis trimera (Less.) DC. & 1.3 & & M-OR \\
\hline Asteraceae & Baccharis vulneraria Baker & 2 & & $\mathrm{M}$ \\
\hline Plantaginaceae & Bacopa monnieri (L.) Pennell & 2 & & OR \\
\hline Fabaceae & Bauhinia forficata Link & 1 & & M-OR \\
\hline Begoniaceae & Begonia cucullata Willd. & 1 & & M-OR \\
\hline
\end{tabular}




\section{Continued}

\begin{tabular}{|c|c|c|c|c|}
\hline Berberidaceae & Berberis laurina Billb. & 1 & & $\mathrm{M}-\mathrm{OR}$ \\
\hline Blechnaceae & Blechnum brasiliense Desv. & 1 & & OR \\
\hline Blechnaceae & Blechnum sp. & 1 & & OR \\
\hline Blechnaceae & Blechnum tabulare (Thunb.) Kuhn & 2 & & OR \\
\hline Myrtaceae & Blepharocalyx salicifolius (Kunth) O. Berg & 1.2 & & M-OR-T \\
\hline Poaceae & Bothriochloa laguroides (DC.) Herter var. laguroides & 3 & & FO-OR \\
\hline Bromeliaceae & Bromelia antiacantha Bertol. & 1 & & OR \\
\hline Poaceae & Bromus catharticus Vahl & 3 & & FO-OR \\
\hline Buddlejaceae & Buddleja thyrsoides Lam. & 1.2 & & $\mathrm{M}-\mathrm{OR}$ \\
\hline Arecaceae & Butia odorata (Barb. Rodr.) Noblick & 1.2 & $\mathrm{P}$ & F-M-OR-Fi \\
\hline Asteraceae & Calea uniflora Less. & 1 & & M \\
\hline Cannaceae & Canna glauca $\mathrm{L}$. & 1.2 & & $\mathrm{M}-\mathrm{OR}$ \\
\hline Sapindaceae & Cardiospermum grandiflorum Sw. & 1 & & M \\
\hline Cyperaceae & Carex spp. & 23 & & OR \\
\hline Salicaceae & Casearia sylvestris Sw. & 1 & & M \\
\hline Cucurbitaceae & Cayaponia bonariensis (Mill.) Mart.Crov. & 1 & & M \\
\hline Cannabaceae & Celtis ehrenbergiana (Klotzsch) Liebm. & 1 & & M-OR-T \\
\hline Cannabaceae & Celtis iguanaea (Jacq.) Sarg. & 1 & & M \\
\hline Apiaceae & Centella asiatica (L.) Urb. & $2,3,4$ & & M-OR \\
\hline Rubiaceae & Cephalanthus glabratus (Spreng.) K. Schum. & 2 & & M-OR \\
\hline Cactaceae & Cereus uruguayanus $\mathrm{R}$. Kiesling & 1 & & OR \\
\hline Solanaceae & Cestrum parqui L’Hér. & 1 & & M \\
\hline Asteraceae & $\begin{array}{c}\text { Chaptalia arechavaletae Hieron. } \\
\text { Chascolytrum poomorphum (J. Presl) Essi, } \\
\text { Longhi-Wagner \& }\end{array}$ & 1 & & OR \\
\hline Poaceae & Souza-Chies & 3 & & FO \\
\hline Poaceae & Chascolytrum subaristatum (Lam.) Desv. & 3 & & FO-OR \\
\hline Asteraceae & Chevreulia sarmentosa (Pers.) S.F. Blake & 1.3 & & OR \\
\hline Rubiaceae & Chiococca alba (L.) C.L. Hitchc. & 1 & & M-OR \\
\hline Sapotaceae & Chrysophyllum gonocarpum (Mart. \& Eichler) Engl. & 1 & & $\mathrm{~F}$ \\
\hline Vitaceae & Cissus palmata Poir. & 1 & & OR \\
\hline Vitaceae & Cissus striata Ruiz \& Pav. & 1.2 & & OR \\
\hline Verbenaceae & Citharexylum montevidense (Spreng.) Moldenke & 1 & & OR-T \\
\hline Cardiopteridaceae & Citronella gongonha (Mart.) R.A. Howard & 1 & & M \\
\hline Ranunculaceae & Clematis montevidensis & 1.3 & & M-OR \\
\hline Linaceae & Cliococca selaginoides (Lam.) C.M. Rogers \& Mildner & 1 & & OR \\
\hline Rhamnaceae & Colletia paradoxa (Spreng.) Escal. & 1 & & M-OR \\
\hline Commelinaceae & Commelina diffusa Burm. f. var. diffusa & 4 & & M \\
\hline Rhamnaceae & Condalia buxifolia Reissek & 1 & & M \\
\hline
\end{tabular}




\section{Continued}

\begin{tabular}{|c|c|c|c|}
\hline Asteraceae & Conyza bonariensis (L.) Cronquist & 1.3 & M-OR \\
\hline Poaceae & Cortaderia selloana (Schult. \& Schult. f.) Asch. \& Graebn. & 2 & M-OR-Fi \\
\hline Asteraceae & Criscia stricta (Spreng.) Katinas & 1 & OR \\
\hline Euphorbiaceae & Croton lanatus Lam. & 1 & OR \\
\hline Euphorbiaceae & Croton sp. & 1 & OR \\
\hline Lythraceae & Cuphea fruticosa Spreng. & 2 & M \\
\hline Lythraceae & Cuphea glutinosa Cham. \& Schltdl. & 2 & M-OR \\
\hline Cyperaceae & Cyperus giganteus $\mathrm{Vahl}$ & 1 & $\mathrm{Fi}$ \\
\hline Cyperaceae & Cyperus sesquiflorus (Torr.) Mattf. \& Kük. ex Kük. & 1.2 & \\
\hline Cyperaceae & Cyperus spp. & 3 & OR \\
\hline Poaceae & Danthonia montevidensis Hack. \& Arechav. & 3 & FO \\
\hline Thymelaeaceae & Daphnopsis racemosa Griseb. & 1.2 & OR-Fi \\
\hline Poaceae & Deyeuxia alba J. Presl & 3 & FO-OR \\
\hline Poaceae & $\begin{array}{c}\text { Deyeuxia viridiflavescens (Poir.) Kunth } \\
\text { Deyeuxia viridiflavescens (Poir.) Kunth var. montevidensis (Nees) }\end{array}$ & 2 & FO-OR \\
\hline Poaceae & Cabrera \& Rúgolo & 3 & FO-OR \\
\hline Convolvulaceae & Dichondra microcalyx (Hallier f.) Fabris & $1,2,3$ & OR \\
\hline Convolvulaceae & Dichondra sericea $\mathrm{Sw}$. & 4 & OR \\
\hline Rhamnaceae & Discaria americana Gillies \& Hook. & 1 & M \\
\hline Sapindaceae & Dodonaea viscosa Jacq. & 1.4 & M-OR \\
\hline Moraceae & Dorstenia brasiliensis Lam. & 1.3 & M \\
\hline Pteridaceae & Doryopteris triphylla (Lam.) Christ & 1 & \\
\hline Droseraceae & Drosera brevifolia Pursh & 2 & OR \\
\hline Bromeliaceae & Dyckia remotiflora Otto \& A. Dietr. & 1 & OR \\
\hline Alismataceae & Echinodorus grandiflorus (Cham. \& Schltdl.) Micheli & 2 & M-OR \\
\hline Asteraceae & Eclipta elliptica DC. & 2.3 & OR \\
\hline Asteraceae & Eclipta prostrata (L.) L. & 4 & M \\
\hline Pontederiaceae & Eichhornia azurea (Sw.) Kunth & 2 & M \\
\hline Pontederiaceae & Eichhornia crassipes (Mart.) Solms & 2 & M \\
\hline Ephedraceae & Ephedra tweediana Fisch. \& C.A. Mey. emend. J.H. Hunz. & 1 & M-OR \\
\hline Equisetaceae & Equisetum giganteum $\mathrm{L}$. & 2.3 & M \\
\hline Poaceae & Eragrostis neesii Trin. & 1 & FO \\
\hline Poaceae & Eragrostis retinens Hack. \& Arechav. & 3 & FO \\
\hline Asteraceae & Erechtites valerianifolius (Link ex Spreng.) Less. ex DC. & 4 & OR \\
\hline Poaceae & Erianthus angustifolius $\mathrm{Nees}$ & 2.3 & OR \\
\hline Apiaceae & Eryngium eburneum Decne. & 1.3 & OR \\
\hline Apiaceae & Eryngium horridum Malme & $1,3,4$ & OR \\
\hline Apiaceae & Eryngium nudicaule Lam. & 3 & M-OR \\
\hline Apiaceae & Eryngium pandanifolium Cham. \& Schltdl. & 2 & M-OR-Fi \\
\hline
\end{tabular}




\section{Continued}

\begin{tabular}{|c|c|c|c|c|}
\hline Apiaceae & Eryngium spp. & 3 & & M \\
\hline Fabaceae & Erythrina crista-galli $\mathrm{L}$. & 1 & & M-OR-T \\
\hline Myrtaceae & Eugenia uruguayensis Cambess. & 1.3 & & OR \\
\hline Asteraceae & Eupatorium tweedianum Hook. \& Arn. & 1 & & OR \\
\hline Euphorbiaceae & Euphorbia rochaensis (Croizat) Alonso Paz \& Marchesi & 2 & $\mathrm{P}$ & \\
\hline Euphorbiaceae & Euphorbia serpens Kunth & 1 & & M \\
\hline Convolvulaceae & Evolvulus sericeus $\mathrm{Sw}$. & 1.3 & & OR \\
\hline Moraceae & Ficus cestrifolia Schott & 1 & $\mathrm{P}$ & OR \\
\hline Moraceae & Ficus luschnathiana (Miq.) Miq. & 1.3 & & F-M-OR \\
\hline Rubiaceae & Galium sp. & 3 & & OR \\
\hline Asteraceae & Gamochaeta americana (Mill.) Wedd. & 3 & & M \\
\hline Asteraceae & Gamochaeta simplicicaulis (Willd. ex Spreng.) Cabrera & 1 & & M \\
\hline Asteraceae & Gamochaeta sp. & 4 & & M-OR \\
\hline Verbenaceae & Glandularia peruviana (L.) Small & 3 & & OR \\
\hline Verbenaceae & Glandularia selloi (Spreng.) Tronc. & 3.4 & & OR \\
\hline Rubiaceae & Guettarda uruguensis Cham. \& Schltdl. & 1 & & OR \\
\hline Orchidaceae & Habenaria parviflora & 4 & & OR \\
\hline Amaryllidaceae & Habranthus gracilifolius Herb. & 3 & & OR \\
\hline Lythraceae & Heimia salicifolia (Kunth) Link & 3 & & M \\
\hline Boraginaceae & Heliotropium curassavicum & 2 & $\mathrm{P}$ & M \\
\hline Iridaceae & Herbertia lahue (Molina) Goldblatt & 3 & & $\mathrm{M}-\mathrm{OR}$ \\
\hline Limnocharitaceae & Hydrocleys nymphoides (Willd.) Buchenau & 2 & & OR \\
\hline Apiaceae & Hydrocotyle bonariensis Lam. & $1,2,3$ & & M-OR \\
\hline Apiaceae & Hydrocotyle ranunculoides L. f. & 2 & & M-OR \\
\hline Hypoxidaceae & Hypoxis decumbens $\mathrm{L}$. & 1.3 & & OR \\
\hline Convolvulaceae & Ipomoea alba L. & 1 & & OR \\
\hline Convolvulaceae & Ipomoea sp. & 1 & & M-OR \\
\hline Poaceae & Ischaemum minus J. Presl & 2.3 & & FO-OR \\
\hline Isoetaceae & Isoëtes weberi Herter & 2 & $\mathrm{P}$ & OR \\
\hline Asteraceae & Jaegeria hirta (Lag.) Less. & 2.3 & & M \\
\hline Malpighiaceae & Janusia guaranitica (A. St.-Hil.) A. Juss. & 1 & & OR \\
\hline Santalaceae & Jodina rhombifolia (Hook. \& Arn.) Reissek & 1 & & M-OR-T \\
\hline Juncaceae & Juncus acutus $\mathrm{L}$. & 2 & & OR \\
\hline Juncaceae & Juncus sp. & 3 & & OR \\
\hline Asteraceae & Jungia floribunda Less. & 1.2 & & M \\
\hline Verbenaceae & Lantana camara L. & 1 & & M-OR \\
\hline Verbenaceae & Lantana megapotamica (Spreng.) Tronc. & 1 & & M-OR \\
\hline Poaceae & Leersia hexandra $S_{W}$. & 1.3 & & FO \\
\hline
\end{tabular}




\section{Continued}

\begin{tabular}{|c|c|c|c|c|}
\hline Lemnaceae & Lemna & 2 & & OR \\
\hline Hydrocharitaceae & Limnobium laevigatum (Humb. \& Bonpl. ex Willd.) Heine & 2 & & M-OR \\
\hline Anacardiaceae & Lithraea brasiliensis Marchand & 1 & & M-OR \\
\hline Anacardiaceae & Lithraea molleoides (Vell.) Engl. & 1 & & M \\
\hline Asteraceae & Lucilia acutifolia (Poir.) Cass. & 1 & & OR \\
\hline Asteraceae & Lucilia nitens Less. & 1 & & OR \\
\hline Onagraceae & Ludwigia caparosa (Cambess.) H. Hara & 2 & & OR \\
\hline Onagraceae & Ludwigia peploides (Kunth) P.H. Raven & 2 & & $\mathrm{M}-\mathrm{OR}$ \\
\hline Poaceae & Luziola peruviana Juss. ex J.F. Gmel. & 2 & & FO \\
\hline Thelypteridaceae & Macrothelypteris torresiana (Gaudich.) Ching & 2 & & \\
\hline Apocynaceae & Mandevilla coccinea (Hook. \& Arn.) Woodson & 1 & & OR \\
\hline Rosaceae & Margyricarpus pinnatus (Lam.) Kuntze & 4 & & $\mathrm{M}-\mathrm{OR}$ \\
\hline Celastraceae & Maytenus ilicifolia Mart. ex Reissek & 1 & & $\mathrm{M}-\mathrm{OR}$ \\
\hline Poaceae & Melica macra Nees & 3 & & OR \\
\hline Poaceae & Melica sarmentosa Nees & 1 & & OR \\
\hline Apocynaceae & Metastelma difussum & 1 & & OR \\
\hline Polypodiaceae & Microgramma squamulosa (Kaulf.) de la Sota & 1 & & M \\
\hline Asteraceae & Mikania micrantha Kunth & 2.4 & & M \\
\hline Poaceae & Mnesithea selloana (Hack.) de Koning \& Sosef & 3 & & FO \\
\hline Asteraceae & Mutisia coccinea A. St.-Hil. & 1 & & OR \\
\hline Myrtaceae & Myrceugenia glaucescens (Cambess.) D. Legrand \& Kausel & 1 & & OR \\
\hline Haloragaceae & Myriophyllum aquaticum (Vell.) Verdc. & 2 & & OR \\
\hline Myrtaceae & Myrrhinium atropurpureum Schott var. octandrum Benth. & 1 & & OR-T \\
\hline Primulaceae & Myrsine coriacea (Sw.) R. Br. ex Roem. \& Schult. & 1 & & OR \\
\hline Primulaceae & Myrsine laetevirens (Mez) Arechav. & 1.2 & & OR \\
\hline Primulaceae & Myrsine parvula (Mez) Otegui & 1 & & \\
\hline Primulaceae & Myrsine umbellata Mart. & 1 & $\mathrm{P}$ & OR \\
\hline Poaceae & Nassella charruana (Arechav.) Barkworth & 3 & & OR \\
\hline Poaceae & Nassella mucronata (Kunth) Pohl & 3 & & $\mathrm{FO}$ \\
\hline Alliaceae & Nothoscordum gramineum (Sims) Beauverd & 1 & & \\
\hline Asteraceae & Noticastrum diffusum (Pers.) Cabrera & 1 & & OR \\
\hline Menyanthaceae & Nymphoides indica (L.) Kuntze & 2 & & M-OR \\
\hline Onagraceae & Oenothera affinis & 4 & & M-OR \\
\hline Onagraceae & Oenothera parodiana Munz & 4 & & OR \\
\hline Poaceae & Oplismenus hirtellus (L.) P. Beauv. & 1 & & OR \\
\hline Cactaceae & Opuntia arechavaletae Speg. & 1 & & OR \\
\hline Osmundaceae & Osmunda spectabilis Willd. & 1 & & OR \\
\hline Oxalidaceae & Oxalis sp. & 1 & & F-M-OR \\
\hline
\end{tabular}




\section{Continued}

\begin{tabular}{|c|c|c|c|c|}
\hline Poaceae & Panicum bergii Arechav. & 3 & & FO \\
\hline Poaceae & Panicum gouinii E. Fourn. & 3 & & FO \\
\hline Poaceae & Panicum prionitis Nees & 2 & & $\mathrm{Fi}$ \\
\hline Poaceae & Panicum racemosum (P. Beauv.) Spreng. & 2 & & OR \\
\hline Poaceae & Panicum sabulorum Lam. & 3 & & $\mathrm{FO}$ \\
\hline Poaceae & Paspalum denticulatum Trin. & 3 & & OR \\
\hline Poaceae & Paspalum dilatatum Poir. & 3 & & FO \\
\hline Poaceae & Paspalum notatum Flüggé & 3 & & FO-OR \\
\hline Poaceae & Paspalum plicatulum Michx. & 3 & & FO-OR \\
\hline Poaceae & Paspalum pumilum Nees & 1.3 & & FO-OR \\
\hline Poaceae & Paspalum quadrifarium Lam. & 2.3 & & OR-Fi \\
\hline Poaceae & Paspalum urvillei & 3 & & FO \\
\hline Poaceae & Paspalum vaginatum $\mathrm{Sw}$. & 2.4 & & OR \\
\hline Passifloraceae & Passiflora caerulea L. & 1 & & F-M-OR \\
\hline Piperaceae & Peperomia catharinae Miq. & 5 & & \\
\hline Solanaceae & Petunia axillaris (Lam.) Britton, Stern \& Poggenb. & 3 & & OR \\
\hline Amaranthaceae & Pfaffia tuberosa (Spreng.) Hicken & 3 & & OR \\
\hline Poaceae & Pharus lappulaceus Aubl. & 1 & $\mathrm{P}$ & OR \\
\hline Verbenaceae & $\begin{array}{c}\text { Phyla nodiflora (L.) Greene var. minor (Gillies \& Hook. ex Hook.) N. } \\
\text { O’Leary \& P. Peralta }\end{array}$ & 3 & & M \\
\hline Phytolaccaceae & Phytolacca dioica & 1 & & M-OR \\
\hline Asteraceae & Picrosia longifolia D. Don & 1.3 & & M \\
\hline Poaceae & Piptochaetium montevidense (Spreng.) Parodi & 3 & & FO-OR \\
\hline Poaceae & Piptochaetium stipoides (Trin. \& Rupr.) Hack. ex Arechav. & 3 & & FO \\
\hline Araceae & Pistia stratiotes $\mathrm{L}$. & 2 & & M-OR \\
\hline Plantaginaceae & Plantago sp. & 1.3 & & M-OR \\
\hline Polypodiaceae & Pleopeltis lepidopteris (Langsd. \& Fisch.) de la Sota & 1 & & OR \\
\hline Asteraceae & Pluchea sagittalis (Lam.) Cabrera & 13 & & M-OR \\
\hline Poaceae & Poa bonariensis (Lam.) Kunth & 3 & & FO-OR \\
\hline Asteraceae & Podocoma notobellidiastrum (Griseb.) G.L. Nesom & 1 & & M \\
\hline Polygalaceae & Polygala linoides Poir. var. linoides & 3 & & M-OR \\
\hline Polygonaceae & Polygonum acuminatum Kunth & 2.4 & & M \\
\hline Polygonaceae & Polygonum punctatum Elliott & 1.2 & & M-OR \\
\hline Poaceae & Polypogon elongatus Kunth & 3 & & M \\
\hline Pontederiaceae & Pontederia cordata $\mathrm{L}$. & 2 & & M \\
\hline Asteraceae & Pseudognaphalium cheiranthifolium (Lam.) Hilliard \& B.L. Burtt & 4 & & M \\
\hline Myrtaceae & Psidium cattleianum Sabine & 1 & $\mathrm{P}$ & F-M-OR \\
\hline Dennstaedtiaceae & Pteridium arachnoideum (Kaulf.) Maxon & 1 & & OR \\
\hline Asteraceae & Pterocaulon lorentzii Malme & 1 & & OR \\
\hline
\end{tabular}




\section{Continued}

\begin{tabular}{|c|c|c|c|c|}
\hline Ranunculaceae & Ranunculus apiifolius Pers. & 2 & & \\
\hline Marsileaceae & Regnellidium diphyllum Lindm. & 2 & & OR \\
\hline Amaryllidaceae & Rhodophiala bifida (Herb.) Traub & 1 & & OR \\
\hline Annonaceae & Rollinia maritima Záchia & 1 & & \\
\hline Dryopteridaceae & Rumohra adiantiformis (G. Forst.) Ching & 1 & & M-OR \\
\hline Alismataceae & Sagittaria montevidensis Cham. \& Schltdl. & 2,4 & & M-OR \\
\hline Salicaceae & Salix humboldtiana Willd. & 1.2 & & M-OR-T \\
\hline Lamiaceae & Salvia guaranitica A. St.-Hil. ex Benth. & 1.2 & & \\
\hline Lamiaceae & Salvia procurrens Benth. & 2 & & OR \\
\hline Salviniaceae & Salvinia sp. & 2 & & M \\
\hline Adoxaceae & Sambucus australis Cham. \& Schltdl. & 1 & & M \\
\hline Euphorbiaceae & Sapium glandulosum (L.) Morong & $1,2,3$ & & OR \\
\hline Anacardiaceae & Schinus engleri F.A. Barkley & 1 & & OR \\
\hline Anacardiaceae & Schinus longifolius (Lindl.) Speg. & 1 & & M-OR \\
\hline Poaceae & Schizachyrium spicatum (Spreng.) Herter & 3 & & OR \\
\hline Cyperaceae & Schoenoplectus americanus (Pers.) Volkart ex Schinz \& R. Keller & 2 & & OR \\
\hline Cyperaceae & Schoenoplectus californicus (C.A. Mey.) Soják & 2 & & M-OR-Fi \\
\hline Cyperaceae & Scirpus giganteus Kunth & 2 & & OR \\
\hline Lamiaceae & Scutellaria racemosa Pers. & 2.3 & & OR \\
\hline Rhamnaceae & Scutia buxifolia Reissek & 1.3 & & M-OR-T \\
\hline Euphorbiaceae & Sebastiania brasiliensis Spreng. & 1.2 & & M \\
\hline Euphorbiaceae & Sebastiania commersoniana (Baill.) L.B. Sm. \& Downs & 1 & & M \\
\hline Asteraceae & Senecio brasiliensis (Spreng.) Less. & 2.3 & & OR \\
\hline Asteraceae & Senecio crassiflorus (Poir.) DC. & 4 & & OR \\
\hline Asteraceae & Senecio montevidensis (Spreng.) Baker & 1 & & OR \\
\hline Asteraceae & Senecio ostenii Mattf. & 4 & $\mathrm{P}$ & OR \\
\hline Asteraceae & Senecio platensis Arechav. & 4 & & OR \\
\hline Asteraceae & Senecio selloi (Spreng.) DC. & 2.4 & & OR \\
\hline Fabaceae & Senna corymbosa (Lam.) H.S. Irwin \& Barneby & 1.2 & & $\mathrm{M}-\mathrm{OR}$ \\
\hline Polypodiaceae & Serpocaulon catharinae (Langsd. \& Fisch.) A.R. Sm. & 1 & & OR \\
\hline Fabaceae & Sesbania punicea (Cav.) Benth. & 2 & & M-OR \\
\hline Poaceae & Setaria parviflora (Poir.) Kerguélen & 1.3 & & FO-OR \\
\hline Malvaceae & Sida rhombifolia L. & 2.3 & & M-OR \\
\hline Sapotaceae & Sideroxylon obtusifolium (Roem. \& Schult.) T.D. Penn. & 1 & $\mathrm{P}$ & M \\
\hline Gesneriaceae & Sinningia allagophylla (Mart.) Wiehler & 1 & & OR \\
\hline Iridaceae & Sisyrinchium platense I.M. Johnst. & 3 & & $\mathrm{M}-\mathrm{OR}$ \\
\hline Smilacaceae & Smilax campestris Griseb. & 1 & & M \\
\hline Solanaceae & Solanum commersonii Dunal ex Poir. ssp. commersonii & 3 & & M-OR \\
\hline
\end{tabular}




\section{Continued}

\begin{tabular}{|c|c|c|c|c|}
\hline Solanaceae & Solanum glaucophyllum Desf. & 2 & & M-OR \\
\hline Solanaceae & Solanum mauritianum Scop. & 2 & & M-OR \\
\hline Solanaceae & Solanum sisymbriifolium Lam. & 3 & & M-OR \\
\hline Asteraceae & Solidago chilensis Meyen var. chilensis & 2 & & M-OR \\
\hline Asteraceae & Sommerfeltia spinulosa (Spreng.) Less. & 1 & $\mathrm{P}$ & OR \\
\hline Poaceae & Sporobolus indicus (L.) R. Br. & 1.3 & & OR \\
\hline Poaceae & Steinchisma hians (Elliott) Nash & 3 & & FO-OR \\
\hline Poaceae & Stenotaphrum secundatum (Walter) Kuntze & 3 & & FO-OR \\
\hline Asteraceae & Stevia satureiifolia (Lam.) Sch. Bip. ex Klotzsch var. satureiifolia & 1 & & M-OR \\
\hline Poaceae & Stipa papposa Nees & 3 & & OR \\
\hline Arecaceae & Syagrus romanzoffiana (Cham.) Glassman & 1 & & OR \\
\hline Asteraceae & Symphyotrichum squamatum (Spreng.) G.L. Nesom & 2 & & OR \\
\hline Asteraceae & Tessaria absinthioides (Hook. \& Arn.) DC. & 2 & & OR \\
\hline Thelypteridaceae & Thelypteris sp. & 1 & & OR \\
\hline Bromeliaceae & Tillandsia aeranthos (Loisel.) L.B. Sm. & 5 & & M-OR \\
\hline Bromeliaceae & Tillandsia usneoides (L.) $L$. & 5 & & M \\
\hline Commelinaceae & Tradescantia fluminensis Vell. & 2 & & M-OR \\
\hline Euphorbiaceae & Tragia volubilis $\mathrm{L}$. & 3 & & M \\
\hline Fabaceae & Trifolium polymorphum Poir. & 3 & & FO-OR \\
\hline Loranthaceae & Tripodanthus acutifolius (Ruiz \& Pav.) Tiegh. & 1,2 & & M-OR \\
\hline Asteraceae & Trixis praestans (Vell.) Cabrera & 1 & & OR \\
\hline Typhaceae & Typha domingensis Pers. & 2 & & M-OR-Fi \\
\hline Typhaceae & Typhasp. & 1.2 & & OR \\
\hline Boraginaceae & Varronia curassavica Jacq. & 1.2 & $\mathrm{P}$ & OR \\
\hline Verbenaceae & Verbena bonariensis $\mathrm{L}$. & 2 & & M-OR \\
\hline Verbenaceae & Verbena intermedia Gillies \& Hook. ex Hook. & 3 & & M \\
\hline Verbenaceae & Verbena montevidensis Spreng. & 1 & & M \\
\hline Fabaceae & Vigna luteola (Jacq.) Benth. & 2 & & FO-OR \\
\hline Poaceae & Vulpia australis (Nees ex Steud.) C.H. Blom & 3 & & OR \\
\hline Campanulaceae & Wahlenbergia linarioides (Lam.) A. DC. & 4 & & OR \\
\hline Lemnaceae & Wolffia brasiliensis Wedd. & 2 & & OR \\
\hline Salicaceae & Xylosma tweediana (Clos) Eichler & 1 & & OR \\
\hline Xyridaceae & Xyris jupicai Rich. & 2 & & M \\
\hline Rutaceae & Zanthoxylum fagara (L.) Sarg. & 1 & & M-OR-T \\
\hline Rutaceae & Zanthoxylum rhoifolium Lam. & 1 & & M-OR \\
\hline Rutaceae & Zanthoxylum sp. & 1 & & \\
\hline Amaryllidaceae & Zephyranthes sp. & 3 & & OR \\
\hline Poaceae & Zizaniopsis bonariensis (Balansa \& Poitr.) Speg. & 2 & & $\mathrm{Fi}$ \\
\hline
\end{tabular}

1: Forests, schrubs and rocky outcrops, F: Food; 2: Wetlands, FO: Fooder; 3: Rangelands, M: Medicinal; 4: Coast, OR: Ornamental; 5: Epiphyte, Fi: Fiber, T: Timber. 
alent to the number of species. It must be noted, however, that the goal was not to complete an exhaustive floristic list but to identify the most relevant species which are structuring the landscape.

The two predominant families found are Poaceae (16.6\% of the taxa) and Asteraceae (16.9\% of the taxa). Such results are consistent with the fact that grasses form, along with asteraceas, the two largest families of Uruguayan and Brazilian natural grasslands (Lezama et al., 2006; Overbeck et al., 2006). The families that come next in number of taxa are Verbenaceae (3.3\%), Fabaceae (3\%), Apiaceae (2.7\%), Euphorbiaceae (2.7\%), Cyperaceae (2.7\%), Myrtaceae (2\%) and Solanaceae $(2 \%)$. The other identified families are represented by 5 or fewer taxa. Among the 302 taxa, we found 11 species that had been previously identified by Marchesi et al. (2013) as of high priority for conservation in Uruguay based on endemism, rarity and limited distribution criteria, in addition to butia palms, which appear on the list of endangered species due to their lack of regeneration (Table 3). In terms of plant genetic resources, species for medicinal (130), ornamental (221), fodder (34), food (8), timber (12) and fiber (10) use were included in the group of 302 taxa (among many other uses). Among plant genetic resources, 106 multipurpose species stand out.

Natural grasslands or "campos" have the highest percentage of occupation in the studied area (Table 2), just as it happens throughout the Uruguayan territory. The geographic location of Uruguay in a subtropical-temperate climate transition zone, the geomorphological and soil diversity and the confluence of different floras have originated a wide diversity of "campos" types (Rosengurtt, 1943; Millot et al., 1987; Lezama et al., 2011), this being a typical feature of the Pampa biome. The natural grasslands of the palm forests are a particular case due to their botanical composition, which differs from that of the surrounding fields (Rivas et al., 2014). Within this context of species and ecosystem diversity, plant genetic resources have evolved, mainly grasses and legumes used for forage, which coexist in a network with herbaceous species used mainly for medicinal and ornamental purposes.

Wetlands, which occupy nearly $13 \%$ of the territory of the palm forest, include both permanently flooded lands and lands which are only flooded at certain times of the year. Among the typical environments, we distinguished marshes with floating and submerged rooted plants, watergrass or gramales, which are herbaceous formations consisting mainly of Luziola peruviana, a variety of monospecific groupings, including those of Scirpus giganteus, Schoenoplectus californicus, Eryngium pandanifolium and E. eburneum, Typha domingensis, Erythrina crista-galli, Juncus acutus, Salix humboldtiana, Cephalanthus glabratus, Solanum glaucophyllum, and panicgrass or pajonales, which are dense communities of Panicum prionitis, Erianthus angustifolia or Paspalum quadrifarium (Alonso, 1998; Rivas, 2013). Acid wetlands are yet another particular case found in this landscape (Alonso \& Bassagoda, 2006). Among the best known plant genetic resources in wetland environments, we distinguish those that are used in construction, furniture making, basketry and handicrafts. 
The proportion of native forests in this landscape is 3 - 4 times higher than the average for Uruguay of around 4\% (Table 2). Different types of forests and scrublands were identified, which are named according to their topographical location: montane, coastal and riverine (Brussa \& Grela 2007). Another very particular community called "ombu forest" (Phytolacca dioica) is found, being the largest grouping of this species in the country, which stretches along $20 \mathrm{ki}$ lometers by the shores of Laguna de Castillos (Castillos Lagoon) (RodríguezGallego, 2006). Among the different plant genetic resources found in the forests, we may highlight those with food, timber, medicinal and ornamental use.

Within this context of ecosystem diversity, coastal communities (Fagúndez \& Lezama, 2005) and vegetation accompanying rocky outcrops are also found.

Palm forests (Figure 3), as a specific type of forest, are mainly located on the middle plains, creating different landscapes not only due to their varying density levels, but also due to the differences in the accompanying vegetation and their associated anthropic uses (Rivas, 2013). Nowadays, butia palms are mainly used
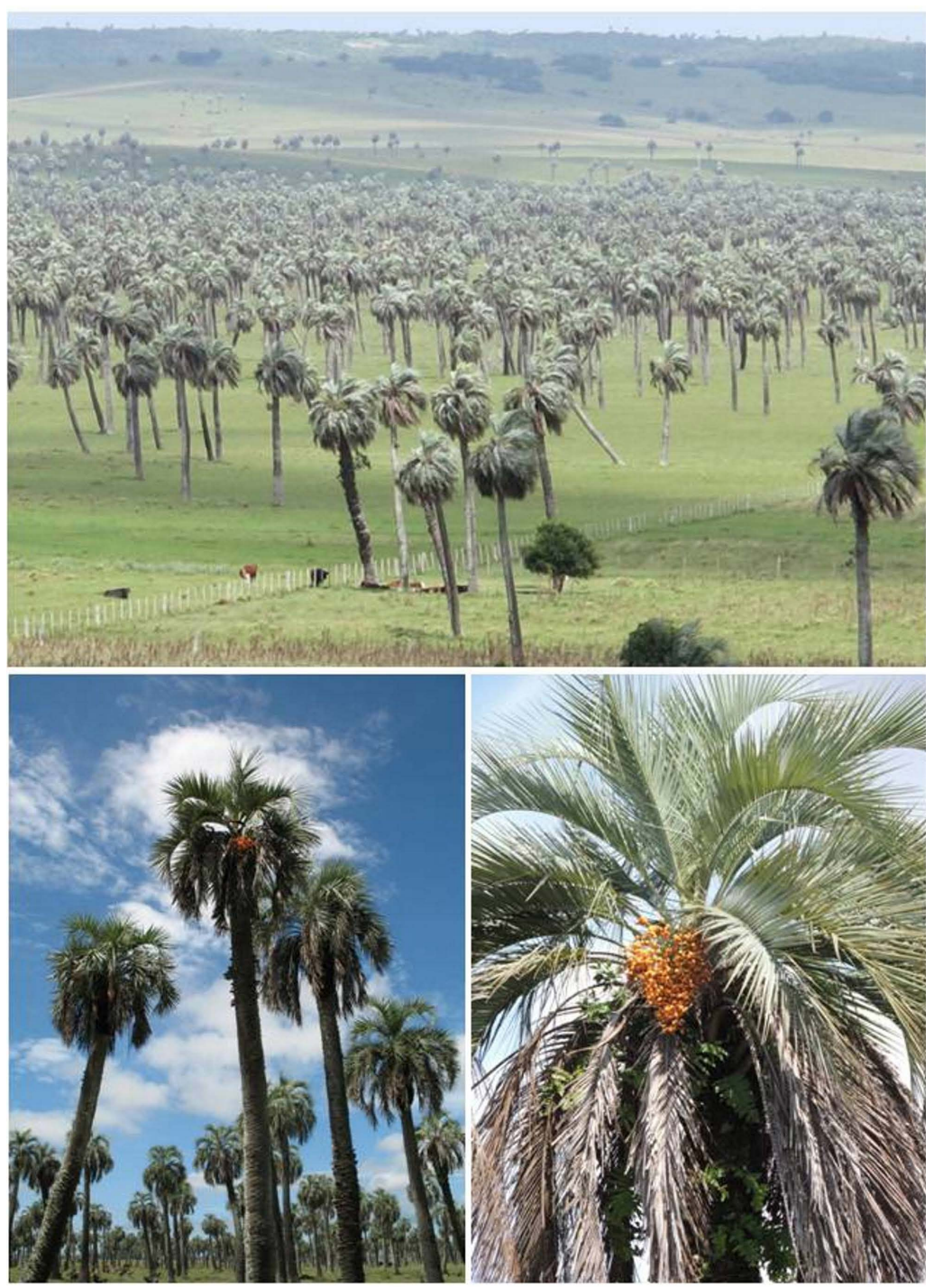

Figure 3. Butia odorata: Palm forest, palm and butia. 
for food. Also, leaf fibers are used in handicrafts and the whole plant is used in landscaping projects with ornamental purposes. Sifted pulp, fiber and seeds of high nutritional value are obtained from fresh fruit products and used in the production of a wide range of foods such as ice cream, dessert sauce, jam and marmalade, sweet and sour sauce, stuffing for chocolates, cookies and pralines (Crosa et al., 2014), in addition to the typical and traditional butia liqueur.

\subsection{Palm Forest Distribution in Agricultural Land}

Within the surveyed area there were a total of 2153 lots, which added up to an area of $158,450.8$ hectares. This number is different to the total area of the surveyed territory (Table 2) as some rural lots are not entirely located within the surveyed area.

There are 700 rural lots where palm forests are found (32.5\%), with varying proportions that range from less than $1 \%$ to $100 \%$ of the total area. Moreover, a relatively low proportion of these lots have a high percentage of palm forest coverage. A total of 117 lots have more than $50 \%$ of their area covered by palm forest that could be considered relevant to the conservation plan. However, in order to effectively determine the importance of these lots in the design of the conservation area, it is necessary to link this information to the area which is indeed occupied by each of them as well as to identify the farms and their location within the general context of distribution of the palm forest and its different density levels.

By comparing the information on lots and the database of owners and tenants who manage cattle we were able to sort into farms $77 \%$ of the cases $(539 / 700$ lots), a relatively satisfactory scenario considering they cover $87 \%$ of the area of the Castillos palm forest.

We managed to configure a total of 212 farms where the palm forest is found, 101 of which have all lots covered by palm forest and 111 of which have lots where the palm forest may or may not be found. Farms where palm forest is found have a size that ranges from 5.15 to 3848 hectares and a modal value of 78 hectares. The majority of them, more specifically $86.8 \%$, have less than 500 hectares, a slightly higher proportion than the average for the department of Rocha (78.5\%) (DIEA, 2013). These farms are considered units of family agriculture according to Uruguayan law, which states that farmers can not exploit an area of more than 500 hectares with CONEAT 100 (an index related to the average production capacity of the country) and they must live within $50 \mathrm{~km}$ of the property, have no more than two employees and that agriculture has to account for their main source of income (MGAP, 2009). The group of farmers with less than 20 hectares (11.3\%) is also slightly above the average for the department of Rocha (9.5\%).

Out of the 10,094.3 hectares of land covered by palm forest, 9241 hectares are located in farms of more than 100 hectares and 7174.11 hectares in farms of more than 500 hectares (Figure 4). The Pearson correlation coefficient between farm size and palm forest area is of 0.70 . This result becomes a crucial element 


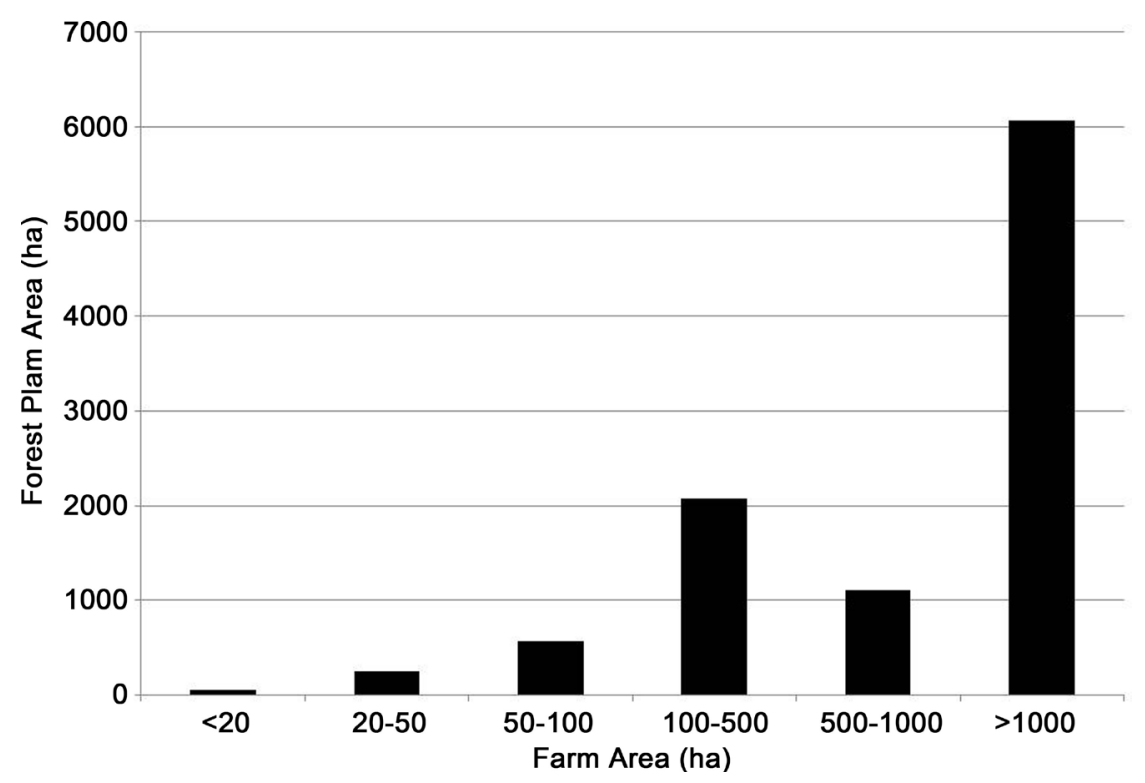

Figure 4. Forest palm area (ha) by size of farms (ha).

for the design of a conservation plan for the palm forest. Larger farms are of higher priority, while in Uruguay policies to support farmers within the framework of environmental conservation and climate change mitigation programs are usually directed at family farmers with smaller farms.

By overlapping layers of farms and palm forest we noticed that 18 farms (Figure 5), all of which are characterized by having more than 100 hectares of palm forest, have a total of 6952.40 hectares of palm forest $(68.9 \%$ of the palm forest is located within farms) and account for $45.5 \%$ of the total area of the farms. This situation had not been previously quantified, so it becomes an important element in the planning and formulation of a management plan for palm forest conservation. These 18 producers own substantial areas of palm forest of very low density, while 6 producers own areas of more than 100 hectares of low density. The importance of these low and very low density palm forests is that, under monitored grazing conditions, regeneration can take place (Rivas \& Barbieri 2015). In the palm forest area with the highest density, hardly any new butia plants can be found under conditions of exclusion or low stocking rates of grazing, probably due to the creation of an environment with greater humidity and less luminosity. Palm forests of medium density are also well represented by all these farmers.

Areas of palm forest with high and very high density levels occupy, in general terms, a relatively low area (Table 1 ) for this group of 18 properties. However, in a second group of farms where there are between 50 and 100 hectares of palm forest, two farms stand out for having areas of dense and very dense palm forest. By including these two farms as part of the proposed conservation area, palm forests of all density levels are represented. It would also promote the development of ecotourism and the marketing of butia by-products, which are particularly found in this area of high aesthetic value and easy access known as "Vuelta del Palmar". 


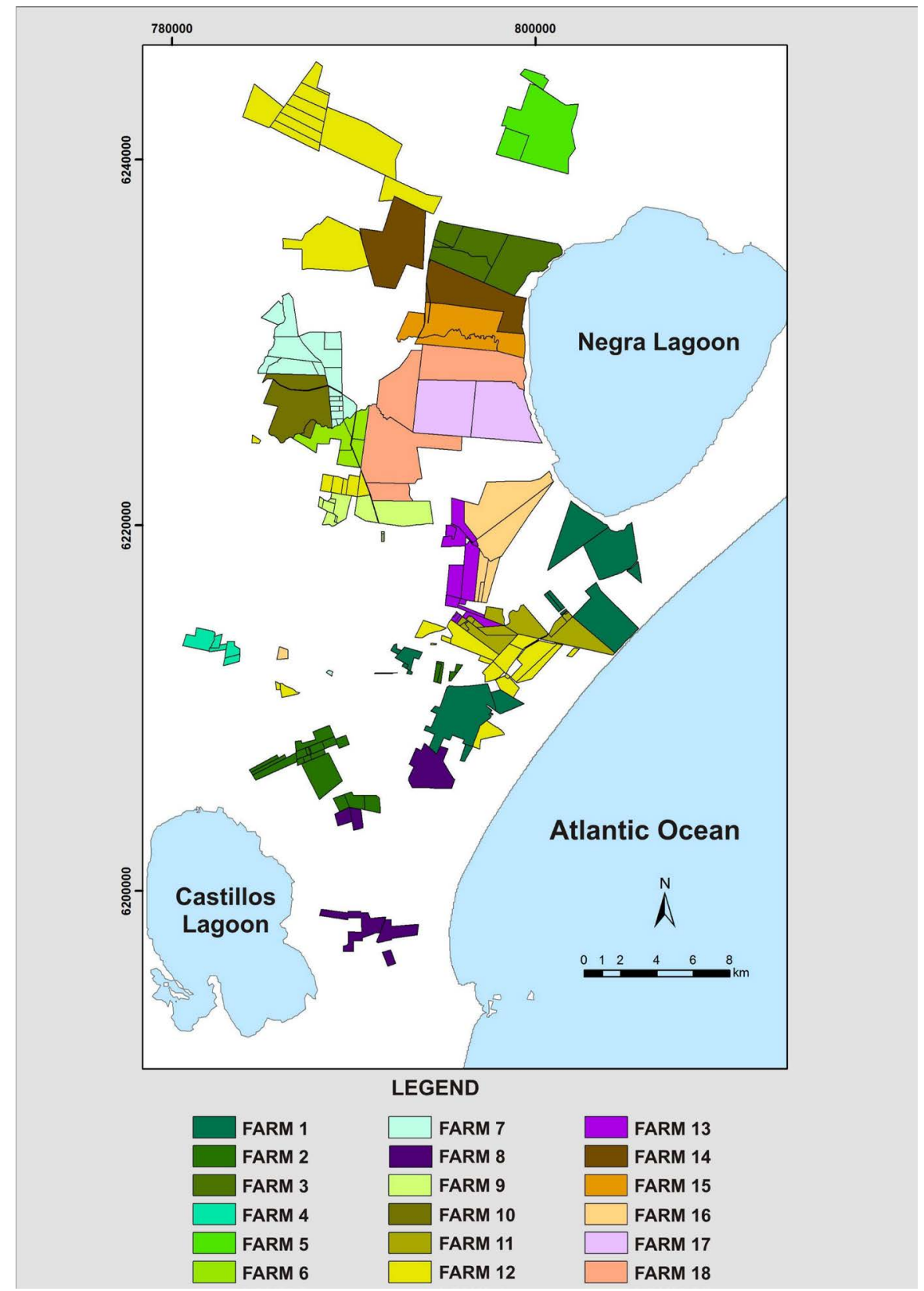

Figure 5. Map showing the location of the 18 priority properties to integrate the conservation area.

Given the need to prioritize some properties, the different density levels of palm forest and/or the strong presence of other plant communities, some factors that would increase the value of conservation proposals are considered. As proposed by Zimmerman \& Runckle (2010), conservation priorities should focus on the representation of plant communities within a context of landscape conservation. In that sense, 5 of these farms have large areas of wetlands, yet another landscape at conservation risk due to the drainage works carried out by some farmers. Coastal forests near Laguna Negra (Black Lagoon) as well as montane forests add an extra value to the palm forest conservation sites.

A reserve comprising these 20 farmers is considered appropriate, taking into 
account the heterogeneity of the environments, the quality of the available habitat, a low edge effect and the concept of minimum viable population (Van Dyke, 2008; Hodgson et al., 2011). Although there are no studies that define the need for a certain population size of butia palms, it would be large enough so as to ensure the conservation of the palm forest. The connectivity of the area, which depends on the potential spread of the species and on habitat fragmentation (Luque et al., 2012), can be considered appropriate, although studies on the spread of pollen and seeds of butia palms are scarce (Rodríguez-Mazzini \& Molina, 2000). Nor is there information on gene flow or on whether palm forests are a single population or they are structured in more subpopulations. The observed fragmentation is mainly explained by natural environmental conditions such as the presence of hills and wetlands, which are unsuitable environments for butia palms.

\section{Conclusion}

The main contributions of this work for the establishment of a conservation area within the butia palm forest of Castillos are the set up of a Geographic Information System (GIS) and the survey of plant communities, species and genetic resources. This GIS allowed us to determine the environmental conditions and the distribution of the palm forest and its density levels in each farm. By analyzing this information, we concluded there are 212 farms in which the palm forest is found, the larger ones having greater proportions of palm forest. The presence in the forest palm landscape of a high ecosystemic, specific and genetic richness values the territory for its conservation. Valuable plant resources with current and potential uses stand out among the 302 surveyed taxa as well as 11 species of high conservation priority apart from butia palms.

As this is an agricultural area that belongs to private owners, the focus of any proposal should be based on the coexistence of production practices with management techniques that can be adapted to conservation strategies and the inclusion of farmers and local dwellers in the definition and management of the area (Bridgewater, 2016). In this sense, the focus of Biosphere Reserves (PROBIDES, 1999; Pezzani, 2007; Ozyavuz \& Yazgan, 2010), some categories of IUCN protected areas such as Protected Landscapes (IUCN, 2008), the Regional Natural Parks (SNAP, 2010) and the GIAHS (Globally Important Agricultural Heritage Systems) (Koohafkan \& Cruz, 2011) are valid options to implement the conservation of Butia palm forests within the framework of integrated biocultural landscape conservation alternatives. Management plans and conservation policies should be framed within a context of sustainable agricultural systems (Dale et al., 2013; Benoit et al., 2012; Baiamonte et al., 2015).

\section{Acknowledgements}

We thank the Sectorial Committee for Scientific Research (CSIC-University of the Republic) and the National Agency for Research and Innovation (ANII) of Uruguay for the financing of various projects that made this work possible. We 
are also grateful to the Federal University of Pelotas (Brazil), Embrapa Temperate Climate (Brazil) and CAPES (Brazilian Agency for the Improvement of Higher Education Personnel) for their valuable cooperation and contribution to the conservation and sustainable use of Butia. We especially thank Martin Jaurena, César Fagúndez, Maria Puppo, Inés Espasandín and Jerónimo Pardiñas for their support in field work, Patricia Mai for her collaboration in the organization of taxonomic information, Federico Condón, Laura del Puerto and Mariana Vilaró for their assistance in writing this paper and the farmers and organizations for their cooperation and permission to have access to their lands.

\section{References}

Agnoletti, M., \& Rotherham, I. D. (2015). Landscape and Biocultural Diversity. Biodiversity and Conservation, 24, 3155-3165. https://doi.org/10.1007/s10531-015-1003-8

Alonso, E. (1998). Aquatic Plants in the Eastern Wetlands. Montevideo: PROBIDES.

Alonso, E., \& Bassagoda, M. J. (2006). Flora and Vegetation along the Uruguayan Atlantic Coast and the River Plate. In R. Menafra, L. Rodríguez-Gallego, F. Scarabino, \& D. Conde (Eds.), In Guidelines for the Conservation and Management of Uruguayan Coastal Areas (pp. 71-88). Montevideo: Vida Silvestre Uruguay (Uruguayan Wildlife).

Baiamonte, G., Domina, G., Raimondo, F. M., \& Bazan, G. (2015). Agricultural Landscapes and Biodiversity Conservation: A Case Study in Sicily (Italy). Biodiversity and Conservation, 24, 3201-3216. https://doi.org/10.1007/s10531-015-0950-4

Benoit, M., Rizzo, D., Marraccini, E., Moonen, A. C., Galli, M., Lardon, S., Rapey, H., Thenail, C., \& Bonari, E. (2012). Landscape Agronomy: A New Field for Addressing Agricultural Landscape Dynamics. Landscape Ecology, 27, 1385-1394. https://doi.org/10.1007/s10980-012-9802-8

Berlingeri, C., \& Crespo, M. B. (2012). Inventory of Related Wild Species of Priority Crops in Venezuela. Genetic Resources and Crop Evolution, 59, 655-681. https://doi.org/10.1007/s10722-011-9709-2

Bridgewater, P. (2016). The Anthropocene Biosphere: Do Threatened Species, Red Lists, and Protected Areas Have a Future Role in Nature Conservation? Biodiversity and Conservation, 25, 603-607. https://doi.org/10.1007/s10531-016-1062-5

Brussa, C. A., \& Grela, I. A. (2007). Uruguayan Tree Flora, Mainly in Rivera and Tacuarembó. Montevideo: COFUSA.

Brussaard, L., Caron, P., Campbell, B., Morgan, B., Lipper, L., Mainka, S., Rabbinge, R., Babin, D., \& Pulleman, M. (2010). Reconciling Biodiversity Conservation and Food Security: Scientific Challenges for a New Agriculture. Current Opinion in Environmental Sustainability, 2, 34-42. https://doi.org/10.1016/j.cosust.2010.03.007

Catalog of Vascular Plants of the Southern Cone (2016). http://www2.darwin.edu.ar/Proyectos/FloraArgentina/fa.htm

Crosa, M. J., Burzaco, P., Irisity, M., Gioscia, D., Sosa, J., \& Ayres, C. (2014). Valorization of Butia Fruits and Their Manufacture. In P. Betancurt, \& M. J. Crosa (Eds.), Native Fruit Appreciation as a Way of Promoting Local Development. Food and Agricultural Exploitation of Butia Palms in Rocha (pp. 25-40). Montevideo: FPTA.

Cullotta, S., \& Maetzke, F. (2009). Forest Management Planning at Different Geographic Levels in Italy: Hierarchy, Current Tools and Ongoing Development. International Forestry Review, 11, 475-489. https://doi.org/10.1505/ifor.11.4.475 
Dabezies, J. M. (2011). Plant Processing, Yesterday and Today: An Approach to Some of the Current Uses of Butia capitata Palms in Order to Understand Some of Its Past Uses. Trama. Revista de Cultura y Patrimonio, 2-3, 10-21.

Dale, V. H., Kline K. L., Kaffka, S. R., \& Langeveld, J. W. A. (2013). A Landscape Perspective on Sustainability of Agricultural Systems. Landscape Ecology, 28, 1111-1123. https://doi.org/10.1007/s10980-012-9814-4

Fagúndez, C., \& Lezama, F. (2005). Spatial Distribution of Coastal Vegetation along the River Plate and the Uruguayan Atlantic Coast. Montevideo: Freplata.

FAO (1996). Global Plan of Action for the Conservation and Sustainable Utilization of Plant Genetic Resources for Food and Agriculture. Rome: FAO.

FAO (2012). Second Global Plan of Action for Plant Genetic Resources for Food and Agriculture. Rome: FAO.

Filippini Alba, J. M., Schroder, V. F., \& Nobrega, M. R. (2012). Land Cover Change Detection in Southern Brazil through Orbital Imagery Classification Methods. In B. Escalante (Ed.), Remote Sensing: Applications (pp. 99-116). Rijeka: Intech. https://doi.org/10.5772/36940

Geymonat, G., \& Rocha, N. (2009). Mbotia; A Unique Ecosystem in the World. Castillos: Casa Ambiental.

Guarino, L. (1995). Mapping the Ecogeographic Distribution of Biodiversity. In L. Guarino, V. Ramanatha Rao, \& R. Reid (Eds.), Collecting Plant Genetic Diversity: Technical Guidelines (pp. 287-314). Roma: IPGRI.

Hawkes, J. G., Maxted, N., \& Zohary, D. (1997). Reserve Design. In N. Maxted, B.V. Ford-Lloyd, \& J.G. Hawkes (Eds.), Plant Genetic Conservation. The in Situ Approach (pp. 132-143). London: Chapman \& Hall.

Hodgson, J. A., Moilanen, A., Wintle, B. A., \& Thomas, C. D. (2011). Habitat Area, Quality and Connectivity: Striking the Balance for Efficient Conservation. Journal of Applied Ecology, 48, 148-152. https://doi.org/10.1111/j.1365-2664.2010.01919.x

IDEuy (2008). Temporary Data Array (Conjunto de Datos Provisorios-CDP). http://descarga-cdp-ide.agesic.gub.uy/

Institute of Agricultural Statistics (DIEA) (2013). General Agricultural Census 2011. Preliminary Report, Montevideo: DIEA.

International Union for Conservation of Nature (IUCN) (2008). Directrices para la aplicación de las categorías de gestión de áreas protegidas. Gland: IUCN.

Iriondo, J. M., Maxted, N., Dulloo, M. E. (2008). Conserving Plant Genetic Diversity in Protected Areas. Population Management of Crop Wild Relatives. London: CAB International. https://doi.org/10.1079/9781845932824.0000

Koohafkan, P., \& de la Cruz, M. J. (2011). Conservation and Adaptive Management of Globally Important Agricultural Heritage Systems (GIAHS). Journal of Resources and Ecology, 2, 22-28.

Lezama, F., Altesor, A., León, R. J., \& Paruelo, J. M. (2006). Vegetation Heterogeneity of Natural Grasslands in the Uruguayan Basaltic Region. Ecología Austral, 16, 167-182.

Lezama, F., Altesor, A., Pereira, M., \& Paruelo, J. M. (2011). Description of Vegetation Heterogeneity of Natural Grasslands in the Main Geomorphologic Regions of Uruguay. In A. Altesor, W. Ayala, \& J. M. Paruelo (Eds.), Ecological and Technological Guidelines for Pasture Management (pp. 15-32). Montevideo: FPTA.

López Mazz, J. M., \& Pintos, S. (2001). The Archaeological Landscape of Laguna Negra (Black Lagoon). In Uruguayan Archaeology towards the Turn of the Millennium (Vol. 1). Montevideo: AVA.

Luque, S., Saura, S., \& Fortin, M. J. (2012). Landscape Connectivity Analysis for Conser- 
vation: Insights from Combining New Methods with Ecological and Genetic Data. Landscape Ecology, 27, 153-157. https://doi.org/10.1007/s10980-011-9700-5

Magos Brehm, J., Maxted, N., Ford-Lloyd, B. V., \& Martins-Louçao, M. A. (2008). Natural Inventories of Crop Wild Relatives and Wild Harvested Plants: Case-Study for Portugal. Genetic Resources and Crop Evolution, 55, 779-796.

https://doi.org/10.1007/s10722-007-9283-9

Marchesi, E., Alonso, E., Delfino, L., García, M., Haretche, F., \& Brussa, C. (2013). Vascular Plants. In A. Soutullo, C. Clavijo, \& J. A. Martínez-Lanfranco (Eds.), Priority Conservation Species in Uruguay. Vertebrates, Continental Mollusks and Vascular Plants (pp. 27-71). Montevideo: SNAP.

Maxted, N., Guarino, L., \& Dulloo, M. E. (1997). Management and Monitoring. In N. Maxted, B. V. Ford-Lloyd, \& J. G. Hawkes (Eds.), Plant Genetic Conservation. The in Situ Approach (pp. 144-159). London: Chapman \& Hall. https://doi.org/10.1007/978-94-009-1437-7

Millot, J. C., Methol, R., \& Risso, D. (1987). Survey on Natural Grasslands and Extensive Improvements in Farming Areas in Uruguay. Montevideo: FUCREA-CHPA.

Ministry of Farming, Agriculture and Fisheries (MGAP) (1976). Classification Chart of Uruguayan Soils. Montevideo: MGAP.

Ministry of Farming, Agriculture and Fisheries (MGAP) (2009). Decree 527/008 of 29/07/09. Defining Family Farmers. Montevideo: MGAP.

Ministry of Farming, Agriculture and Fisheries (MGAP), \& Institute of Natural Resources (2013). http://www.renare.gub.uy

National Network of Protected Areas (SNAP) (2010). Protected Areas and Natural Regional Parks. An Insight to the Uruguayan Context. Discussion Document. Strengthening the Implementation Process of the National Network of Protected Areas. Project. Montevideo: SNAP.

Overbeck, G. E., Muller, S. C., Pillar, V. D., \& Pfadenhauer, J. (2006). Floristic Composition, Environmental Variation and Species Distribution Patterns in Burned Grassland in Southern Brazil. Brazilian Journal of Biology, 66, 1073-1090. https://doi.org/10.1590/S1519-69842006000600015

Ozyavuz, M., \& Yazgan, M. E. (2010). Planning of Igneada Longos (Flooded) Forests as a Biosphere Reserve. Journal of Coastal Research, 26, 1104-1111. https://doi.org/10.2112/JCOASTRES-D-09-00065.1

Parra-Quijano, M., Iriondo, J. M., \& Torres, E. (2012). Ecogeographical Land Characterization Maps as a Tool for Assessing Plant Adaptation and Their Implications in Agrobiodiversity Studies. Genetic Resources and Crop Evolution, 59, 205-217. https://doi.org/10.1007/s10722-011-9676-7

Perrino, P., Laghetti, G., \& Terzi, M. (2006). Modern Concepts for the Sustainable Use of Plant Genetic Resources in the Mediterranean Natural Protected Areas: The Case Study of the Alta Murgia Park (Italy). Genetic Resources and Crop Evolution, 53, 695-710. https://doi.org/10.1007/s10722-004-3942-x

Pezzani, F. (2007). Biosphere Reserve of the Eastern Wetlands, Uruguay. Work Document No. 37, Montevideo: PROBIDES, South-South Cooperation Plan, UNESCO.

PROBIDES (1995). Palm Forests, Palms and Butiá. Didactic Index 4. Montevideo: PROBIDES.

PROBIDES (1999). Management Plan. Biosphere Reserve of the Eastern Wetlands/Uruguay. Montevideo: PROBIDES.

Rajpurohit, D., \& Jang, T. (2015). In Situ and ex Situ Conservation of Plant Genetic Resources and Traditional Knowledge. In R. K. Salgotra, \& B. B. Gupta (Eds.), Plant Ge- 
netic Resources and Traditional Knowledge for Food Security (pp. 137-161). Berlin: Springer. https://doi.org/10.1007/978-981-10-0060-7_8

Reyers, B., O'Farrell, P. J., Nel, J. L., \& Wilson, K. (2012). Expanding the Conservation Toolbox: Conservation Planning of Multifunctional Landscapes. Landscape Ecology, 27, 1121-1134. https://doi.org/10.1007/s10980-012-9761-0

Rivas, M. (2005). Challenges and Alternatives for the in Situ Conservation of Butia capitata Palm Forests (Mart.) Becc. Agrociencia, 9, 161-168.

Rivas, M. (2013). Conservation and Sustainable Utilization of Butia odorata Palm Forests (Barb. Rodr.) Noblick. Doctorate Thesis, Pelotas: Pelotas Federal University.

Rivas, M., \& Barbieri, R. L. (2015). Good Practices for the Sustainable Management of Butia Palm Forests. Brasilia: Embrapa.

Rivas, M., \& Condón, F. (2016). Plant Domestication and Utilization: The Case of the Pampa Biome. In J. M. Al-Khayri, S. M. Jain, \& D. V. Johnson (Eds.), Advances in Plant Breeding Strategies: Breeding, Biotechnology and Molecular Tools (pp. 3-24, Vol. 1). Cham: Springer International Publishing.

Rivas, M., Clausen, A., \&, León-Lobos, P. (2010). In Situ Conservation of Plant Genetic Resources of Importance for Food and Agriculture. In Strategies on Plant Genetic Resources for the Southern Cone Countries (pp. 59-74). Montevideo: IICA-PROCISUR.

Rivas, M., Jaurena, M., Gutiérrez, L., \& Barbieri, R. L. (2014). Plant Diversity of Natural Butia odorata Grasslands (Barb. Rodr.) Noblick in Uruguay. Agrociencia, 18, 14-27.

Rodríguez-Gallego, M. G. (2006). Structure and Regeneration of Ombú Forests (Phytolacca dioica) in Laguna de Castillos (Castillos Lagoon) (Rocha, Uruguay). In R. Menafra, L. Rodríguez-Gallego, F. Scarabino, \& D. Conde (Eds.), Guidelines for the Conservation and Management of Uruguayan Coastal Areas (pp. 503-511). Montevideo: Vida Silvestre Uruguay.

Rodríguez-Mazzini, R., \& Molina, B. (2000). The Forest Fox (Cerdocyon thous) as a Palm Seed Spreading Agent. Work Document No. 30, Rocha: PROBIDES.

Rosengurtt, B. (1943). Studies on Uruguayan Natural Grasslands: Third Contribution. Montevideo: Barreiro y Ramos.

Taylor Lovell, S., DeSantis, S., Nathan, C. A., Olson, M. B., Méndez, V. E., Kominami, H. C., Erickson, D. L., Morris, K. S., \& Morris, W. B. (2010). Integrating Agroecology and Landscape Multifunctionality in Vermont: An Evolving Framework to Evaluate the Design of Agroecosystems. Agricultural Systems, 103, 327-341.

https://doi.org/10.1016/j.agsy.2010.03.003

United Nations (1992). Convention on Biological Diversity.

United Nations (2002). The Global Strategy for Plant Conservation. La Haya: GSPC, CDB.

Van Dyke, F. (2008). The Conservation of Habitat and Landscape. In F. Van Dyke (Ed.), Conservation Biology. Foundations, Concepts, Applications (pp. 279-311, 2nd ed.). Berlin/Heidelberg: Springer Science and Business Media. https://doi.org/10.1007/978-1-4020-6891-1_10

Zaffaroni, C., Rivas, M., Resnichenko, Y., \& Hernández, J. (2005) A Contribution for the Conservation of Unique Landscapes: Butia capitata Palm Forests (Mart.) Becc., in the Department of Rocha, Uruguay. In 10th Annual Report-Conference of Latin American Geographers (pp. 116611-16622). Sao Paulo: EGAL.

Zimmerman, C. L., \& Runkle, J. R. (2010). Using Ecological Land Units for Conservation Planning in a Southwestern Ohio Watershed. Natural Areas Journal, 30, 27-38.

https://doi.org/10.3375/043.030.0104 
Submit or recommend next manuscript to SCIRP and we will provide best service for you:

Accepting pre-submission inquiries through Email, Facebook, LinkedIn, Twitter, etc. A wide selection of journals (inclusive of 9 subjects, more than 200 journals)

Providing 24-hour high-quality service

User-friendly online submission system

Fair and swift peer-review system

Efficient typesetting and proofreading procedure

Display of the result of downloads and visits, as well as the number of cited articles Maximum dissemination of your research work

Submit your manuscript at: http://papersubmission.scirp.org/

Or contact ojf@scirp.org 